\title{
The Carnegie Hubble Program: From parallaxes to the Tully-Fisher relation
}

\author{
V. Scowcroft, ${ }^{1}$ W. L. Freedman, ${ }^{1}$ B. F. Madore,${ }^{1}$ A. Monson, ${ }^{1}$ \\ S. E. Persson, ${ }^{1}$ M. Seibert, ${ }^{1}$ C. Burns, ${ }^{1}$ and J. R. Rigby ${ }^{2}$ \\ ${ }^{1}$ Observatories of the Carnegie Institute of Washington, 813 Santa Barbara Street, Pasadena, \\ CA 91101, USA \\ email: vs@obs.carnegiescience.edu \\ ${ }^{2}$ Observational Cosmology Lab, NASA Goddard Space Flight Center, Greenbelt, MD 20771, \\ USA
}

\begin{abstract}
The Carnegie Hubble Program (CHP) is a Warm Spitzer program with the aim of reducing the uncertainty in the Hubble constant to below $3 \%$. The program is calibrated using Galactic Cepheids with precise parallax distances from the Hubble Space Telescope (HST), combined with a large sample of Cepheids in the Large Magellanic Cloud. We extend the Cepheid distance scale to the Local Group and beyond, into the regime probed by the Tully-Fisher relation. The entire program - from Galactic Cepheids to the most distant galaxies - uses the Spitzer/IRAC instrument. Completing the entire program with a single instrument on a single telescope virtually eliminates instrumental effects, whilst moving to the mid-infrared drastically reduces the reddening and metallicity effects that trouble the optical Cepheid distance scale. Our first measurement of the Hubble constant, using only two CHP galaxies tied into the HST Key Project results has produced a measurement of $\mathrm{H}_{0}=74.3 \pm 2.1$ (systematic) $\mathrm{km} \mathrm{s}^{-1} \mathrm{Mpc}^{-1}$, which corresponds to a systematic uncertainty of $2.8 \%$.
\end{abstract}

Keywords. Cepheids, galaxies: distances and redshifts, distance scale

\section{Introduction}

The Carnegie Hubble Program (CHP) can be summarized in a single sentence: We aim to measure the Hubble constant to an accuracy better than 3\% using the Warm Spitzer mission and the James Webb Space Telescope (JWST). A complete description of the program can be found in Freedman et al. (2011).

The best answer to the question as to why we must continue to work on the accuracy of the Hubble constant can be found in Walker (2012): "The Hubble constant at any given time has always been known to 10 percent, despite having changed over that period by a factor of 10." Although we have resolved the factor-of-two controversy that plagued the field just over a decade ago, we still must drill down further. To quote $\mathrm{Hu}$ (2005): "The single most important complement to the CMB [cosmic microwave background] for measuring the dark energy equation of state at $z \sim 0.5$ is a determination of the Hubble constant to better than a few percent."

The factor-of-two controversy was resolved by the $\mathrm{H}_{0}$ Key Project (Freedman et al. 2001), which brought the uncertainty in $\mathrm{H}_{0}$ down to $10 \%$. The study was performed at optical wavelengths and was anchored by the Cepheid distance to the Large Magellanic Cloud (LMC), which ultimately dominated the systematic error budget. At the time, the distance to the LMC was only known to \pm 0.1 mag. The effect of metallicity on optical Cepheid distances was (and still is) controversial, and since the LMC has a much lower metallicity than the other galaxies in the program, the metallicity effect added 
another large systematic uncertainty to the budget. Reddening is significant at optical wavelengths, leading to increased uncertainties. Finally, instrumental effects, such as the tie-in of the Hubble Space Telescope (HST) to ground-based measurements contribute a large fraction of the error budget. Luckily, over the past decade we have been able to resolve many of these issues.

Great advances were made by Riess et al. (2011), who achieved a precision of $3 \%$ using $H$-band observations with HST. By moving to the near-infrared, they reduced the effects of reddening and metallicity, and also reduced the uncertainty in their distance scale zero point by combining three anchor galaxies (the Milky Way, LMC, and NGC 4258). The Riess et al. study and the CHP complement each other; both use a distance-ladder technique, but study different wavelengths with different instruments, enabling meaningful discussions about systematic effects in both studies.

\section{Warm Spitzer Program}

The CHP observations are conducted using a single instrument on a single telescope, the IRAC camera on Spitzer. We were awarded approximately 1000 hours as part of the post-cryogenic mission, and the observations were all taken between September 2009 and August 2011.

The program is split into six sections:

(a) Galactic Cepheids: 37 Cepheids, including those with parallax measurements from the HST Fine Guidance Sensors, used to calibrate the zero point of the Cepheid Leavitt law (also known as the period-luminosity relation).

(b) LMC Cepheids: 82 Cepheids spread across the LMC used to determine the slope of the Leavitt law.

(c) Local Group: 14 galaxies in the Local Group with known Cepheid populations.

(d) Beyond the Local Group: nine galaxies beyond the Local Group with known Cepheid populations, including the maser host galaxy NGC 4258 and the supernova host M101.

(e) Tully-Fisher sample: 404 galaxies with rotational velocity data available to measure the Tully-Fisher (TF) relation.

(f) Supernova hosts: 44 supernova host galaxies observed by the Carnegie Supernova Project.

The sections move from the closest to most distant objects, employing different measurement techniques as we move further out. The program was designed so that several objects can be used in more than one section, for example M33 will have a Cepheid distance and is used as a TF-relation calibrator. By taking all images for every technique using a single instrument, we have removed the instrumental systematics that were present in the HST Key Project from the tie-in to ground-based observations.

\section{Initial CHP measurement of the Hubble constant}

\subsection{Galactic Cepheids}

The CHP mid-infrared distance ladder is firmly anchored in our own Galaxy (for a full description, see Monson et al. 2012). Using a Galactic calibration, as opposed to one based on observations in the LMC, reduces the possibility of metallicity effects on the zero point of the Leavitt law, since the metallicity of the Milky Way is very similar to that of the other galaxies in our sample, whereas the LMC's metallicity is much lower.

The CHP observing strategy for Milky Way and LMC Cepheids is different from other Cepheid surveys in that we used deterministic sampling. Each Cepheid in these two 

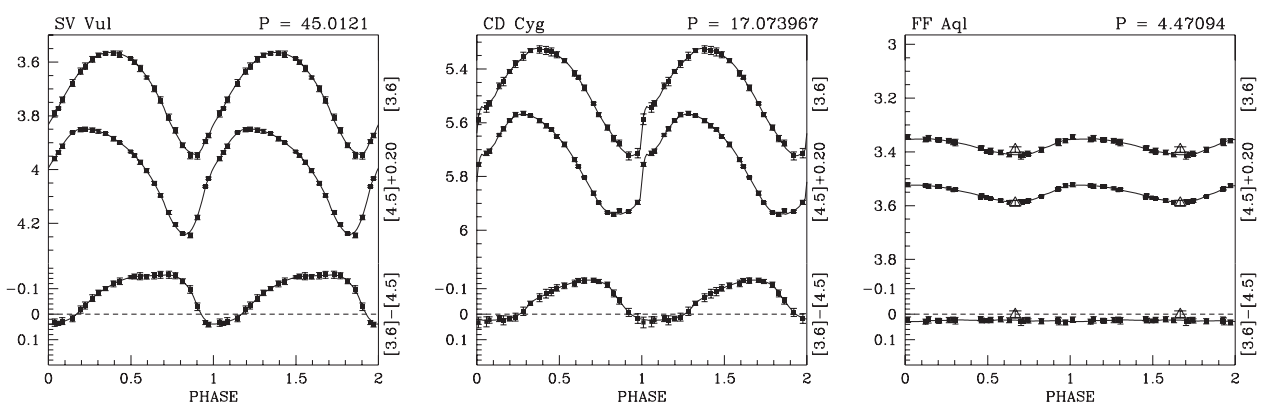

Figure 1. Light curves for three Galactic Cepheids observed using deterministic sampling. From top to bottom, the curves represent [3.6], [4.5], and the [3.6] - [4.5] color. The open triangles represent data from Marengo et al. (2010) when available. Light curves for the whole sample are available in Monson et al. (2012).

samples was observed at 24 regularly spaced points through a single pulsation cycle, producing exquisite light curves with well-defined features in a small fraction of the time that would have been required had the sampling been random. Examples of the Galactic Cepheid light curves are shown in Fig. 1.

The Leavitt law zero point was determined using a subsample of the Galactic Cepheids that had parallax measurements from the HST Fine Guidance Sensors (Benedict et al. 2007). Fig. 2 shows the [3.6] and [4.5] fits. The slopes were fixed to the values measured from the LMC sample (described in Section 3.2) and the zero points were fitted using a least-squares technique. Note, however, that when the slopes were measured using the Milky Way sample, their values were entirely consistent. The LMC slopes were chosen, since they are defined on the basis of a larger sample, and hence have smaller associated uncertainties.

\subsection{LMC Cepheids}

The 82 Cepheids in the LMC were observed identically to the Galactic Cepheids, i.e. using 24 observations equally spaced over a single pulsation cycle. The slope of the Leavitt law was measured using a least-squares fit, excluding Cepheids with periods outside the range $6 \leqslant P \leqslant 60$ days. The short-period Cepheids are excluded, since there is still some debate as to whether the relation is linear all the way to the shortest periods. The longest-period Cepheids were excluded because Cepheids with $P \geqslant 100$ days are known to deviate significantly from the linear relation. We chose 60 days as a cut off, since we did not observe any Cepheids with $60 \leqslant P \leqslant 100$ days. A full description of the LMC program is given by Scowcroft et al. (2011).

\subsection{The effect of $C O$ at $4.5 \mu \mathrm{m}$}

The bottom curves in Fig. 1 represent the [3.6] - [4.5] color throughout the Cepheid's pulsation cycle. Initially we expected the color to be flat and approximately zero. However, a clear color curve emerged for the Milky Way and LMC Cepheids, changing in amplitude and mean color depending on the period of the Cepheid.

The cyclical color change is induced by a CO bandhead situated at $4.6 \mu \mathrm{m}$, directly in the [4.5] IRAC bandpass. It was first discussed in the context of Cepheids by Marengo et al. (2010), who detected its effects from single observations of Milky Way Cepheids, where it appeared as an unexpectedly large scatter in the period-color relation. They modeled the feature, showing that its depth should change as the Cepheid changes temperature throughout its pulsation cycle. 


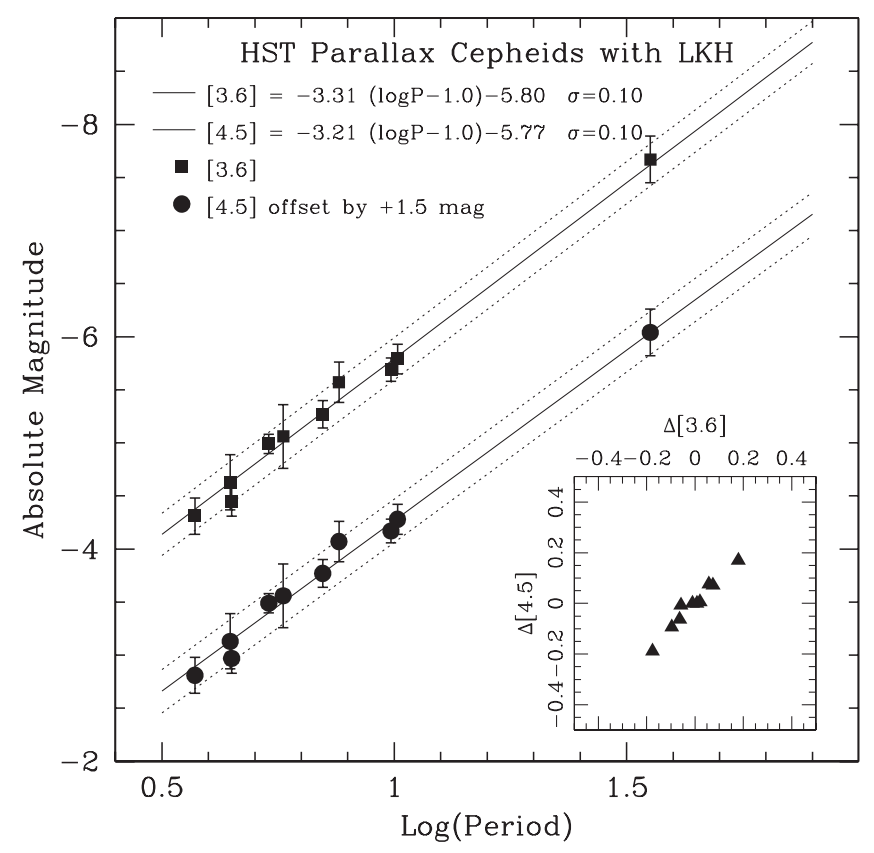

Figure 2. Mid-infrared Leavitt laws for Galactic calibrator Cepheids, from Monson et al. (2012). The slopes correspond to the values derived from the LMC sample (see Section 3.2); identical values (with larger uncertainties) were found when fitted to the Galactic sample. Dotted lines represent $\pm 2 \sigma$ boundaries around the relations.

The CHP color curves are the first measurement of this change through the cycle. The color term behaves as expected in the presence of CO, removing flux from the [4.5] band as the star cools, but leaving the [3.6] band unaffected. For this reason, we have excluded the [4.5] results from our distance measurements and use only the [3.6] measurements to calibrate the Leavitt law and measure Cepheid distances.

\subsection{Distance to the $L M C$}

The distance to the LMC was determined by combining the Milky Way and LMC samples, offsetting the Milky Way sample until the dispersion of all targets around the LMC Leavitt law was minimized, as shown in Fig. 3. This procedure results in a distance modulus of $18.477 \pm 0.033 \mathrm{mag}(49.6 \mathrm{kpc})$. This is consistent with other recent Cepheid measurements (e.g., Benedict et al. 2007; Riess et al. 2011). Walker (2012) compiled distance measurements of the LMC using five independent distance indicators, including Cepheids, RR Lyrae stars, eclipsing binaries, red variables, and red-clump stars. The resulting average distance modulus was $18.48 \mathrm{mag}$, with a full range of $0.1 \mathrm{mag}$, i.e., $\sigma \sim 1.5 \%$, which is in excellent agreement with our result.

\subsection{Mid-infrared calibration of the Hubble constant}

The mid-infrared calibration of the Hubble constant was obtained by adopting the new LMC distance into the $\mathrm{H}_{0}$ Key Project distance ladder and adapting the analysis of systematics accordingly.

Moving to the mid-infrared greatly reduces the systematic uncertainty associated with extinction, which no longer dominates the error budget. By using the parallax Cepheids for the zero-point calibration, the corresponding systematic uncertainty drops to $1.7 \%$. The systematic effect owing to metallicity is dramatically reduced to $1.4 \%$, since 


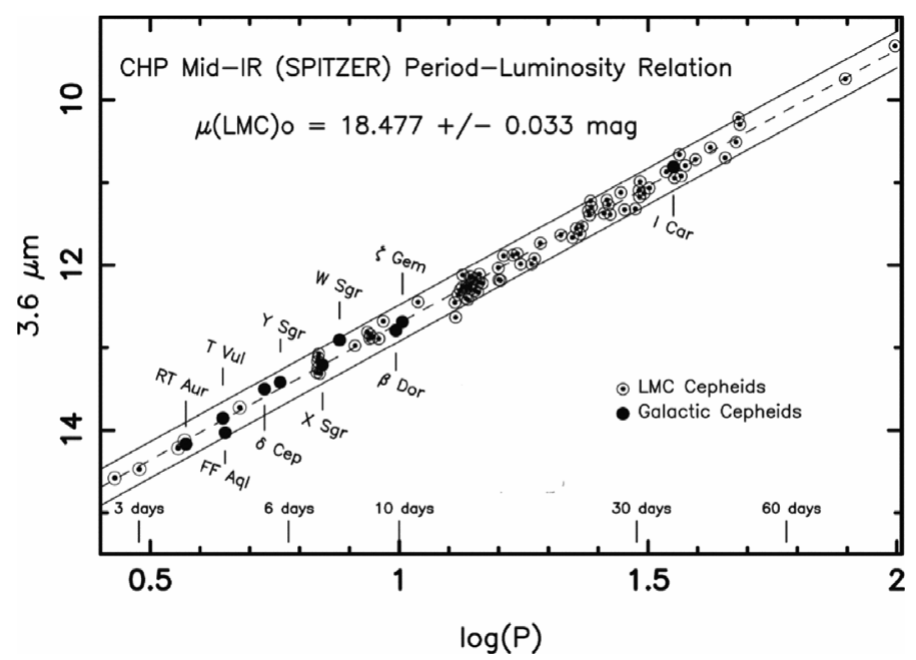

Figure 3. Leavitt law at $3.6 \mu \mathrm{m}$ for $80 \mathrm{LMC}$ Cepheids and 10 Milky Way Cepheids with HST trigonometric parallaxes. Small circled points are LMC Cepheids; large filled circles, individually named, are Galactic Cepheids with trigonometric-parallax measurements. The dashed slope is defined by the sample of 80 LMC stars; the solid lines are $2 \sigma$ ridge lines. Taken from Freedman et al. (2012).

metallicity has no measurable effect at $3.6 \mu \mathrm{m}$. The uncertainties arising from the WFPC2 zero point, crowding, and large-scale flows were reanalyzed and now all lie at $1 \%$.

Combining these values and calculating the statistical uncertainty gives $\mathrm{H}_{0}=74.3 \pm$ 1.5 ( stat.) \pm 2.1 ( syst.) $\mathrm{km} \mathrm{s}^{-1} \mathrm{Mpc}^{-1}$. This value is consistent with the recent measurement by Riess et al. (2011), who calculated $\mathrm{H}_{0}$ in the $H$ band. Full details of our analysis for the LMC distance and Hubble constant calibration can be found in Freedman et al. (2012).

\section{Moving into the Local Group}

The CHP measurement of the Hubble constant will be improved in the near future with revised $3.6 \mu \mathrm{m}$ Cepheid distances to other galaxies in the Local Group. One of the first of these will be IC 1613, a dwarf galaxy situated above the plane of the Milky Way at a distance modulus of 24.33 mag (slightly closer than M33).

Using Spitzer mid-infrared observations combined with near-infrared observations from FourStar (Persson et al. 2012) and archival optical data, we measured the average $E(B-V)$ of the galaxy and computed its distance modulus. The result is shown in Fig. 4.

Using archival optical data in combination with our infrared observations from FouRSTAR and IRAC, we can obtain extinction-corrected distances to all galaxies in our sample. These will then be adopted into the Cepheid portion of the distance ladder and used in the local calibration of the TF relation.

\section{Mid-Infrared Tully-Fisher Relation}

Our work on the TF relation has been performed in collaboration with the Cosmic Flows with Spitzer (CFS) program. Descriptions of the data reduction can be found in Sorce et al. (2012a). 


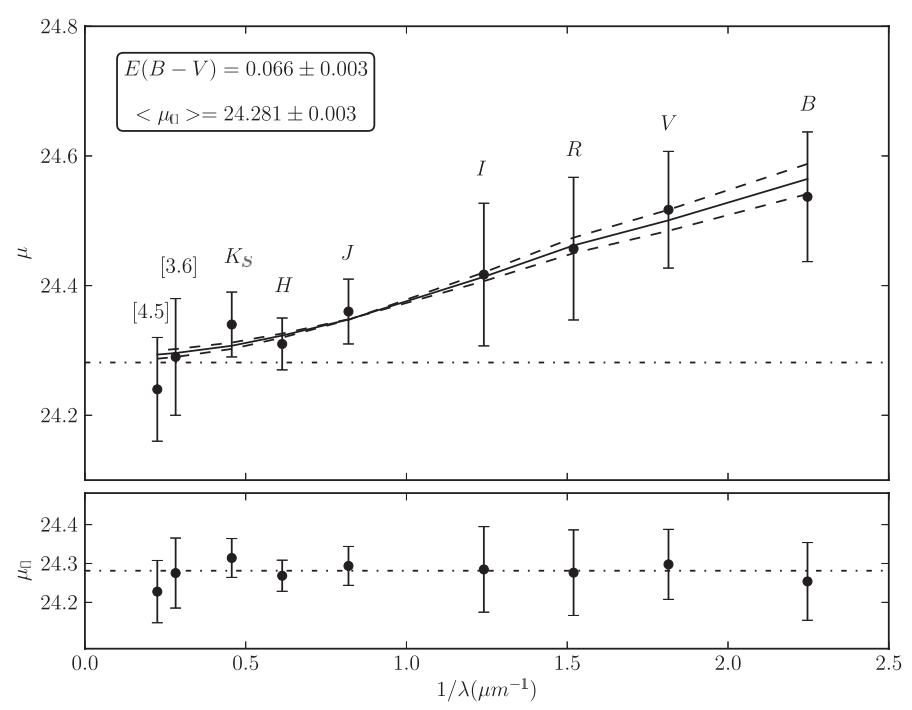

Figure 4. Fitting the IC 1613 distance moduli to the reddening laws of Cardelli et al. (1989)-B to $K_{\mathrm{s}}$-and Indebetouw et al. $(2005), K_{\mathrm{s}}$ to [4.5]. Points at $B$ and $R$ are taken from Freedman (1988), $V$ and $I$ are from Udalski et al. (2001), $J, H$, and $K_{s}$ are our FourStar data, and [3.6] and [4.5] are our IRAC data. The solid line is the best-fitting reddening law, the dashed lines are $\pm 1 \sigma$ around the law, and the dot-dashed line is the resulting reddening-corrected distance modulus. The bottom panel shows the residuals of the extinction-corrected distance moduli around the mean value. From V. Scowcroft et al., in prep.

The mid-infrared TF relation was calibrated assuming the updated LMC distance given in Section 3.4. Local galaxies were used to calibrate the slope and zero point of the $3.6 \mu \mathrm{m}$ relation. It was then applied to several clusters at distances out to $\sim 110 \mathrm{Mpc}$.

Unfortunately, moving from the optical to mid-infrared did not have the same effect on the TF relation as it did on the Leavitt law. The dispersion in the TF relation at 3.6 $\mu \mathrm{m}$ is slightly larger than in the $I$ band. The dispersion of the relation can be reduced by including a color term in the calibration, but it does not reach the width of the $I$-band relation. We believe that the increased dispersion in the mid-infrared may be caused by the population of asymptotic giant branch (AGB) stars that dominate the spectral-energy distribution at these wavelengths.

In Sorce et al. (2012b) we calculate the Hubble parameter for the seven most distant clusters in our sample. Averaging these seven values gives $\mathrm{H}_{0}=73.8 \pm 1.1$ ( stat.) \pm 3.5 ( syst.) $\mathrm{km} \mathrm{s}^{-1} \mathrm{Mpc}^{-1}$. This is consistent with the value obtained in Freedman et al. (2012).

\subsection{Improvements to the Tully-Fisher relation}

There is still room for improvement in the CHP-CFS mid-infrared TF relation. The local calibrators in Sorce et al. (2012b) have been observed as part of the CHP and will soon have updated mid-infrared Cepheid distances. Using the CHP distances will put the calibrator distances on the same instrumental system as the rest of the sample, reducing the systematic uncertainties in the TF distance scale and the resulting $\mathrm{H}_{0}$ measurement.

We must also investigate why the $3.6 \mu \mathrm{m}$ TF relation underperforms relative to the $I$ band. We are currently investigating whether the increase in dispersion in the midinfrared can be explained by the contribution of AGB stars, as is the case in the nearinfrared (Melbourne et al. 2011). 


\section{The Future: $\mathrm{H}_{0}$ to $1 \%$}

The CHP is designed to achieve an accuracy of 3\% using Spitzer and ultimately 2\% with JWST. One might think that $2 \%$ would be good enough, but plans are already afoot to achieve an accuracy of $1 \%$ (Suyu et al. 2012).

A $1 \%$ Hubble constant is needed to further constrain cosmological models. For example, the uncertainty in the dark energy equation-of-state parameter, $w$, is approximately double the uncertainty in $\mathrm{H}_{0}$. A $3 \%$ Hubble constant means a $6 \%$ accuracy in $w$; reducing the uncertainty in $\mathrm{H}_{0}$ to $1 \%$ will bring down the uncertainty in $w$ dramatically to $2 \%$. Further constraining $w$ will allow more accurate predictions of quantities such as the mass and number of relativistic particles.

The biggest improvements will come when we can observe galaxies that contain multiple distance indicators. Fig. 1 of Zaritsky et al. (2012) is an excellent summary of the current situation. The majority of galaxies only have a single distance indicator. If Cepheids are observable, then RR Lyrae are probably too faint, and the galaxy probably is not a supernova host. There are very few galaxies with both geometric distances (from masers or eclipsing binaries) and luminosity distances (for example, from variable stars).

Finally, improvements in theoretical models of Cepheids and supernovae will help us to understand the systematic errors in our measurements, such as metallicity effects.

\section{Summary}

The CHP has made great progress over its first three years. We have measured the distance to the LMC using the mid-infrared Cepheid Leavitt law, finding a value of $\mu=18.477 \pm 0.033 \mathrm{mag}$. The distance is consistent with the most recent measurements from Cepheids as well as other distance indicators.

The distance was applied to the $\mathrm{H}_{0}$ Key Project distance ladder, which was recalibrated in the mid-infrared. We find a local Hubble constant of $\mathrm{H}_{0}=74.3 \pm 1.5$ ( stat.) \pm 2.1 ( syst.) $\mathrm{km} \mathrm{s}^{-1} \mathrm{Mpc}^{-1}$, consistent with the value found by Riess et al. (2011).

Finally, we applied the new LMC distance to the calibrators of the mid-infrared TF relation and calculated the distance to 12 galaxy clusters out to $110 \mathrm{Mpc}$. Using the seven most distant of these, we find a value of $\mathrm{H}_{0}=73.8 \pm 1.1$ ( stat.) \pm 3.5 ( syst.) $\mathrm{km}$ $\mathrm{s}^{-1} \mathrm{Mpc}^{-1}$, consistent with our previous measurement.

In conclusion, the mid-infrared distance scale is in excellent shape. With improvements to Cepheid theory, the TF calibration, and JWST, we may soon break the $1 \%$ barrier for $\mathrm{H}_{0}$.

\section{References}

Benedict, G. F., McArthur, B. E., Feast, M. W., et al. 2007, AJ, 133, 1810

Cardelli, J. A., Clayton, G. C., \& Mathis, J. S. 1989, ApJ, 345, 245

Freedman, W. L. 1988, ApJ, 326, 691

Freedman, W. L., Madore, B. F., Gibson, B. K., et al. 2001, ApJ, 553, 47

Freedman, W. L., Madore, B. F., Scowcroft, V., et al. 2011, AJ, 142, 192

Freedman, W. L., Madore, B. F., Scowcroft, V., Burns, C., Monson, A., Persson, S. E., Seibert, M., \& Rigby, J. R. 2012, ApJ, 758, 24

Hu, W. 2005, in: Observing Dark Energy (Wolff, S.C., \& Lauer, T.R., eds.), Astron. Soc. Pac. Conf. Ser., 339, p. 215

Indebetouw, R., Mathis, J. S., \& Babler, B. L., et al. 2005, ApJ, 619, 931

Marengo, M., Evans, N. R., Barmby, P., Bono, G., Welch, D. L., \& Romaniello, M. 2010, ApJ, 709, 120 
Melbourne, J., Williams, B., Dalcanton, J., Girardi, L., \& Marigo, P. 2011, in: Why Galaxies Care about AGB Stars II: Shining Examples and Common Inhabitants (Kerschbaum, F., Lebzelter, T., \& Wing, R.F., eds.), San Francisco: Astron. Soc. Pac., p. 515

Monson, A. J., Freedman, W. L., Madore, B. F., Persson, S. E., Scowcroft, V., Seibert, M., \& Rigby, J. R. 2012, ApJ, 759, 146

Persson, S. E., Murphy, D., Smee, S., et al. 2012, PASP, submitted

Riess, A. G., Macri, L., Casertano, S. et al. 2011, ApJ, 730, 119

Scowcroft, V., Freedman, W. L., Madore, B. F., Monson, A. J., Persson, S. E., Seibert, M., Rigby, J. R., \& Sturch, L. 2011, ApJ, 743, 76

Sorce, J. G., Courtois, H. M., \& Tully, R. B. 2012a, AJ, 144, 133

Sorce, J. G., Courtois, H. M., Tully, R. B., et al. 2012b, ApJ, submitted

Suyu, S. H., Treu, T., Blandford, R. D., et al. 2012, in: KIPAC workshop on the Hubble constant (arXiv:1202.4459)

Udalski, A., Wyrzykowski, L., Pietrzynski, G., Szewczyk, O., Szymanski, M., Kubiak, M., Soszynski, I., \& Zebrun, K. 2001, Acta Astron., 51, 221

Walker, A. 2012, Ap\&SSS, 341, 43

Zaritsky, D., Zabludoff, A. I., \& Gonzales, A. H. 2012, ApJ, 748, 15 